\title{
The relationship between leukemia and TP53 gene codon Arg72Pro polymorphism: analysis in a multi-ethnic population
}

\author{
Emmanuel Kwateng Drokow1 (i), Yuqing Chen², Hafiz Abdul Waqas Ahmed², Timothy \\ Bonney Oppong ${ }^{3}$, Gloria Selorm Akpabla ${ }^{4}$, Yanru Pei ${ }^{2}$, Maame Awoyoe Kumah, Enyonam \\ Adjoa Neku ${ }^{6}$ \& Kai Sun*,2 \\ ${ }^{1}$ Department of Radiation Oncology, Zhengzhou University People's Hospital \& Henan Provincial People's Hospital Henan, 450003 \\ Zhengzhou, PR China \\ 2Department of Haematology, Zhengzhou University People's Hospital \& Henan Provincial People's Hospital Henan, 450003 \\ Zhengzhou, PR China \\ ${ }^{3}$ Department of Epidemiology \& Biostatistics, College of Public Health, Zhengzhou University, 450001 Zhengzhou, Henan, PR China \\ ${ }^{4}$ Department of Internal Medicine, Tianjin Medical University, 300070 Tianjin, PR China \\ ${ }^{5}$ Department of Internal Medicine, University of Ghana Medical School, KB 77 Korle Bu, Accra, Ghana \\ ${ }^{6}$ Department of Pharmacy, Zhengzhou University, 450001 Zhengzhou, Henan, PR China \\ *Author for correspondence: Tel.: +861823711 0038; sunkai@cellscience.org
}

\begin{abstract}
Aim: Many studies have analyzed the relationship between Arg72Pro polymorphism of TP53 and leukemia; nevertheless, the findings continue to be indeterminate. We, therefore, performed an updated metaanalysis in multi-ethnic groups using specialized software for genome-wide association studies metaanalysis. Materials \& methods: PubMed, EMBASE and Google Scholar were searched up to October 2018. An odds ratio (OR) with the corresponding $95 \% \mathrm{Cl}$ was used to evaluate the strength in the association. Results: This meta-analysis included 16 studies with 2337 cases and 9494 controls. In the overall population, significant relationship between Arg72Pro polymorphism of TP53 and leukemia susceptibility was found in two genetic models (recessive model: $\mathrm{OR}=1.276,95 \% \mathrm{Cl}=1.102-1.476 ; \mathrm{p}=0.01$; overdominant model: $\mathrm{OR}=0.891,95 \% \mathrm{Cl}=0.802-0.988 ; \mathrm{p}=0.03)$. In stratified studies with ethnicity, a significant association was found in five ethnic groups, including Chinese, Americans, Africans, Japanese and Indians. Conclusion: We demonstrated that an association exist between leukemia risk and TP53 gene codon Arg72Pro polymorphism in the recessive and overdominant genetic models. Also, our findings show that the TP53 Arg72Pro polymorphism may influence leukemia development in different populations.
\end{abstract}

First draft submitted: 2 December 2019; Accepted for publication: 26 March 2020; Published online: 17 April 2020

Keywords: genes $\bullet$ leukemia $\bullet$ meta-analysis $\bullet$ polymorphism • TP53

Leukemia is a hematopoietic system malignant progressive disease with abnormal or an increased number of immature leucocytes produced by the bone marrow and other blood-forming organs [1-4]. This suppresses normal blood cell production, resulting in thrombocytopenia, anemia and other clinical syndromes (maladies). The overall prevalence rate of leukemia continues to increase because of its vast and complex features resulting in the poor prognosis of its patients [5,6]. The four major types of leukemia based on cytogenetic analysis are chronic myeloid leukemia, chronic lymphocytic leukemia, acute lymphocytic leukemia and acute myeloid leukemia [7-9]. Several risk factors such as exposure to ionizing radiation, use of cytotoxic drugs, Fanconi anemia, chromosome 21q disorders and genetic variations have been associated with the development of leukemia [10].

The $p 53$, also known as tumor protein or TP53 is a gene which codes for a protein that regulates the cell cycle and therefore acts as a tumor suppressor gene [11,12]. P53 has been portrayed (demonstrated) as 'the guardian of the genome', because of its significant function in 'conserving stability by preventing genome mutation'. The $p 53$ gene is mapped on the chromosome $17(17 \mathrm{p} 13)$ and functions by regulating growth arrest (preventing damaged DNA replication), apoptosis and DNA repair [13]. It also acts as a transcriptional activator in the regulation of

Future Medicine 
Mdm2 expression and the genes involved (engaged) in DNA repairs, apoptosis and growth arrest. In general, the mutation of $p 53$ genes is believed to be related to carcinogenesis. Several pieces of research have demonstrated that TP53 played a crucial role in leukemia development $[11,13]$. The TP53 germline mutations contribute to LiFraumeni Syndrome, a condition in which patients are predisposed to several forms of cancer, such as lymphoma, sarcomas and leukemia [14,15]. Again, the key dysfunctional role for TP53 in leukemogenesis has been demonstrated in Li-Fraumeni Syndrome, through proteomics, large-scale sequencings, preclinical and clinical studies [16-22]. Additionally, the relationship of this gene polymorphism has also been described in preleukemia disorders such as myelodysplastic syndrome [23,24]. The popular and most widely studied polymorphism in cancer is Arg72Pro polymorphism of TP53 at codon 72 of exon 4 . The exchange of guanine to cytosine $(G>C)$ nucleotides related to this polymorphism results in a nonsynonymous change of amino acids from arginine (Arg) to proline (Pro) [13]. The TP53 72Arg variant shows an increased capacity to bring about programmed cell death, while the TP53 72 Pro variant brings about the arrest of the cell cycle more efficiently [25,26]. Bergamaschi et al. revealed that there was a more frequent occurrence of the allele A1 (proline residue, Pro72) in leukemia patients than in healthy individuals [27].

Nevertheless, subsequent investigations indicated distinctive outcomes about TP53 Arg72Pro polymorphism and the susceptibility of leukemia. To date, many scientific studies have examined the relationship between Arg72Pro TP53 polymorphism and leukemia predisposition, but the impact of Arg72Pro TP53 polymorphism in leukemia remains paradoxical because of the inconsistent conclusions in previous studies. The possible reasons for this discrepancies and inconsistencies may be due to ethnicity, different DNA source, sample size and the differences in environmental exposure.

In our quest to increase clarity, we carried out this meta-analysis among different ethnic groups to analyze the possible effect of TP53 codon 72 polymorphism on leukemia susceptibility using a specialized software for genome-wide association studies meta-analysis.

\section{Materials \& methods}

Identification \& eligibility of relevant studies

We searched Google Scholar, EMBASE and PubMed databases for the possible relevant scientific articles up to October 2018 using the following keywords: 'P53 or TP53', 'leukemia' and 'variant or polymorphism'. Furthermore, we involved other relevant scientific articles discovered through manual searching.

\section{Exclusion \& inclusion criteria}

The inclusion criteria of our studies included: cohort or case-control studies, studies assessing the relationship between the risk of leukemia and Arg72Pro polymorphism of TP53, articles with the distribution of the control genotype in Hardy-Weinberg equilibrium and the studies with adequate information for estimating odds ratios (ORs) and 95\% CIs. The exclusion criteria for exclusion studies included: studies with incomplete data, the studies which are not case-control study and duplication of past publication.

\section{Extraction of data}

Two reviewers (Kwateng and Chen) independently extracted the data by following the standardized protocol and procedure for data extraction. Another investigator (Waqas) also participated in the extraction of data. Any disagreements and discrepancies were resolved through full discussion with the third reviewer. The extracted information involved: the year of publication, the name of the first author, cases and controls genotype distribution, ethnicity, country of origin, p-value for Hardy-Weinberg equilibrium, the control source, sample sizes and methods for genotyping.

\section{Quality score evaluation}

Two reviewers independently evaluated the studies qualities in accordance with the quality assessment scale. The cancer genetic factors and epidemiological factors were the basis for these scores. Any dispute between the reviewers was settled through dialogue. The range for the total score was between 0 (low) and 15 (high).

\section{Statistical analysis}

Pooled OR and 95\% CI were used in evaluating the strength of the association between Arg72Pro polymorphism of TP53 and leukemia. The genotypes and alleles were compared using five different genetic models' allele contrast 
model (C vs G), dominant model (CC + GC vs GG), recessive model (CC vs GC + GG), overdominant (CG vs $\mathrm{CC}+\mathrm{GG})$, as well as homozygote (CC vs GG) models. Hardy-Weinberg equilibrium was estimated in the various control arm for the individual study using the $\chi^{2}$ test and the $\mathrm{p}$-value less than $0.05(\mathrm{p}<0.05)$ was considered as the level of significance. The heterogeneity between the studies was evaluated by index (I2). A high value of I2 (>50\%) indicated heterogeneity. If heterogeneity was not present (I2 <50\%), a fixed-effect model was applied for analysis; otherwise, a random-effect model was adopted. The outcomes of the overall OR for all the included studies under the five genetic models were presented using forest plots. We conducted subgroup analyses based on ethnicities, control source and method of genotype. Analysis of sensitivity was carried out to ascertain the impact of individual data sets on the combined estimates. Egger's test and Begg's funnel plot were used in assessing the bias in the publication [28,29]. The web tool META-Genyo, version 12.0 was used in all our analyses [30]. MetaGenyo provides a detailed and complete workflow, which can be implemented without programming skills in an easier-touse setting. In addition, MetaGenyo was designed to guide users via the key stages of a genetic association studies meta-analysis, covering subgroup analysis, heterogeneity test, Hardy-Weinberg analysis, analysis of publication bias, statistical relationship for the various genetic models and robustness evaluation of the outcomes [30].

\section{Results}

\section{Characteristics of the included studies}

A total of 115 articles were identified through the electronic database search $(n=113)$ and other sources $(n=2)$. After 20 duplicates were removed, 95 records were left for further screening. After that, 62 records were excluded because they did not meet our criteria for inclusion; thus, the excluded articles were not associated with Arg72Pro polymorphism of TP53 and leukemia. Full text of the remaining 33 articles was read to evaluate the eligibility and 17 were excluded further due to incomplete data. Finally, our quantitative analysis included 16 records. A total of 18 case-control articles that evaluated the relationship of leukemia with Arg72Pro polymorphism of TP53 were involved in our analysis. The flow diagram for the included studies is shown in (Figure 1). Two of the authors presented two studies each. Hence the sum of 18 case-control studies from 16 articles. Of the 18 studies, four were in Chinese ethnicity group, four in Indian ethnicity group, four in Caucasian ethnicity group, two in Japanese ethnicity group, two in American ethnicity group, one in the African and one in a mixed ethnicity group. There were four hospital-based studies and 14 population-based studies. The characteristics of the studies included are shown in (Table 1) [27-43]. A total of 2337 cases and 9494 controls were investigated.

\section{Subgroup analysis}

We examined the relationship between the Arg72Pro polymorphism of TP53 and leukemia susceptibility in a multi-ethnic population by determining the OR with its 95\% CI under these five genetic models: the homozygote model (CC vs GG), the allele model (C vs G), the recessive model (CC vs GC + GG), the dominant model (CC $+\mathrm{GC}$ vs GG) and overdominant model (CG vs $\mathrm{CC}+\mathrm{GG}$ ). Table 2 shows the results of our subgroup analysis in the multi-ethnic population under the five genetic models. We observed a significant relationship between the Arg72Pro polymorphism of TP53 and leukemia susceptibility in two of the genetic models (recessive model: $\mathrm{OR}=1.276,95 \% \mathrm{CI}=1.102-1.476 ; \mathrm{p}=0.01$; overdominant model: $\mathrm{OR}=0.891,95 \% \mathrm{CI}=0.802-0.988$; $\mathrm{p}=0.03$ ) while no significate relationship was noticed in the other three genetic model (allele model OR $=1.064$, $95 \% \mathrm{CI}=0.973-1.208 ; \mathrm{p}=0.34$; homozygote model $\mathrm{OR}=1.204,95 \% \mathrm{CI}=0.927-1.563 ; \mathrm{p}=0.16$; dominant model OR $=1.002,95 \% \mathrm{CI}=0.843-1.119 ; \mathrm{p}=0.99$; Figures 2-6).

In subgroup analysis by ethnicity, we observed a significant relationship between Arg72Pro polymorphism of TP53 and the risk of leukemia among Chinese (CC vs GG: OR: 1.400, 95\% CI: 1.152-2.085; p = 0.004), Japanese (CC vs GG: $\mathrm{OR}=0.608,95 \% \mathrm{CI}: 0.372-0.993 ; \mathrm{p}=0.041)$ and mixed populations (CC vs GG: OR: 5.396, 95\% CI: 1.033-28.189; $\mathrm{p}=0.045)$ with both homozygote model and allele model. Furthermore, there was also a significate association among Indians (CC + CG vs GG: OR: 0.719, 95\% CI: 0.570-0.907; p = 0.005), Americans (CC + CG vs GG: OR: 1.413, 95\% CI: 1.041-1.917; p = 0.026) and mixed population (CC + CG vs GG: OR: 2.204, 95\% CI: 1.032-4.700; $\mathrm{p}=0.16)$ in dominant model whiles a significate association was observed among the African population in overdominant model (CG vs CC + GG: OR: 0.409, 95\% CI: 0.183-0.913; p = 0.029). No significant association between TP53 Arg72Pro polymorphism and leukemia was observed when the analysis was stratified by source of control (Table 2). 


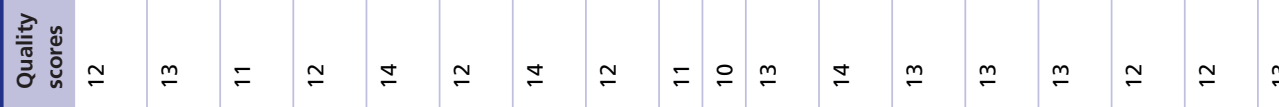

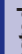

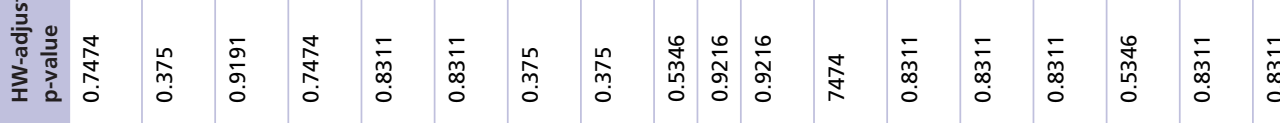

$\frac{20}{\frac{2}{20}}$

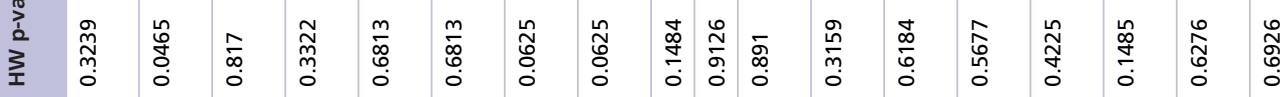

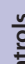

莺

苟 㝵 n

$\frac{n}{2}$

岇

竞

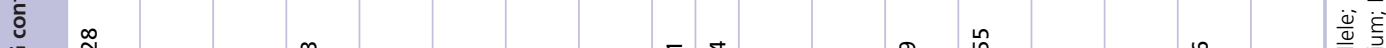

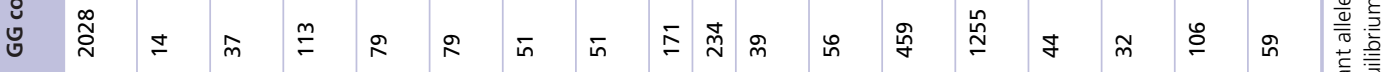

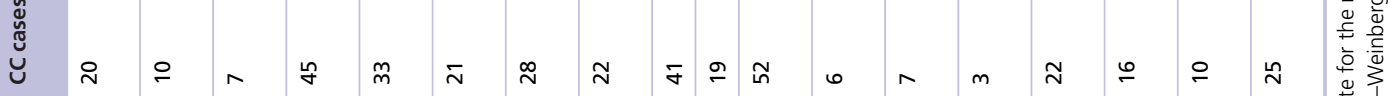

ע

若

பำ

幽

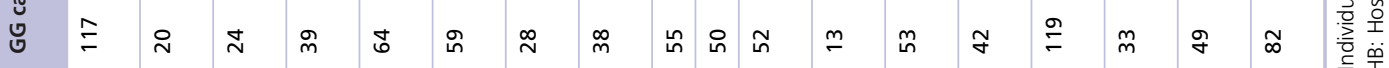

官

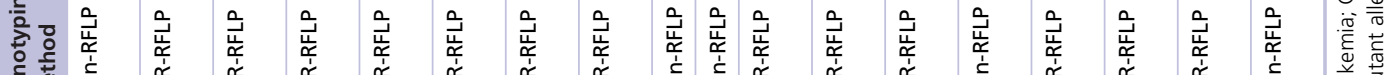

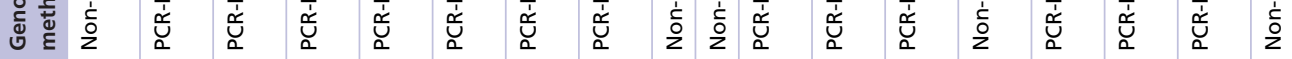




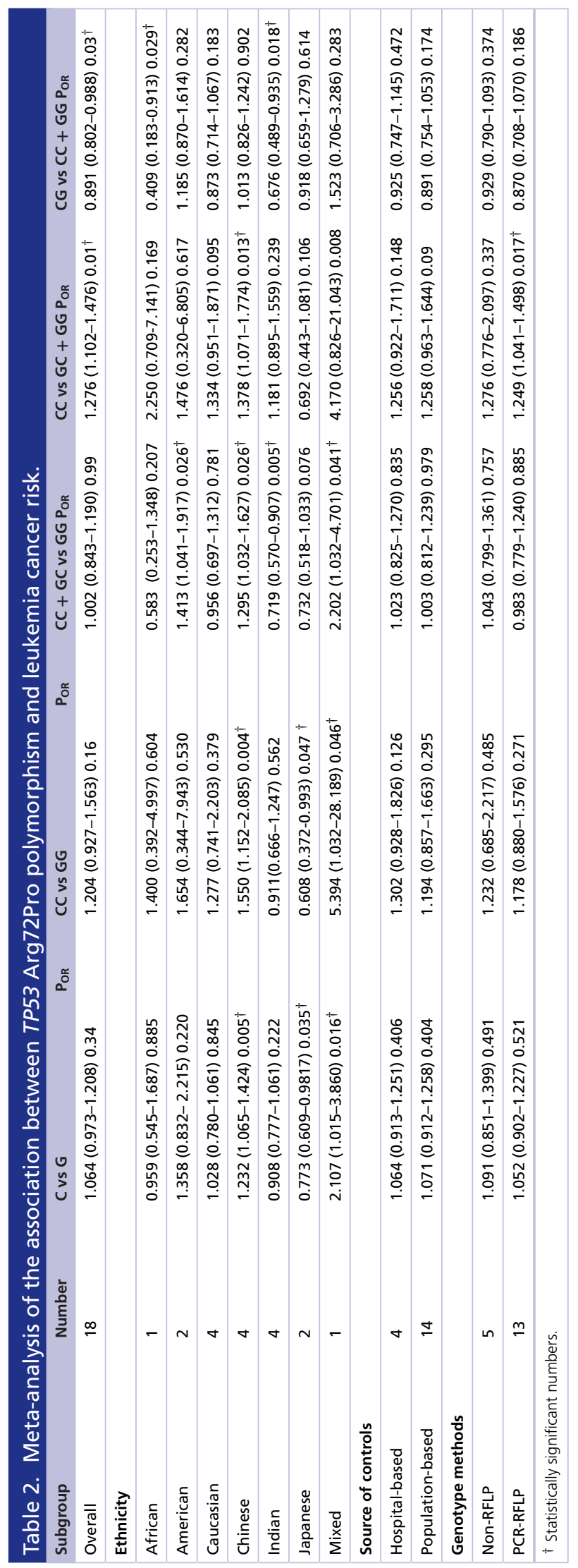




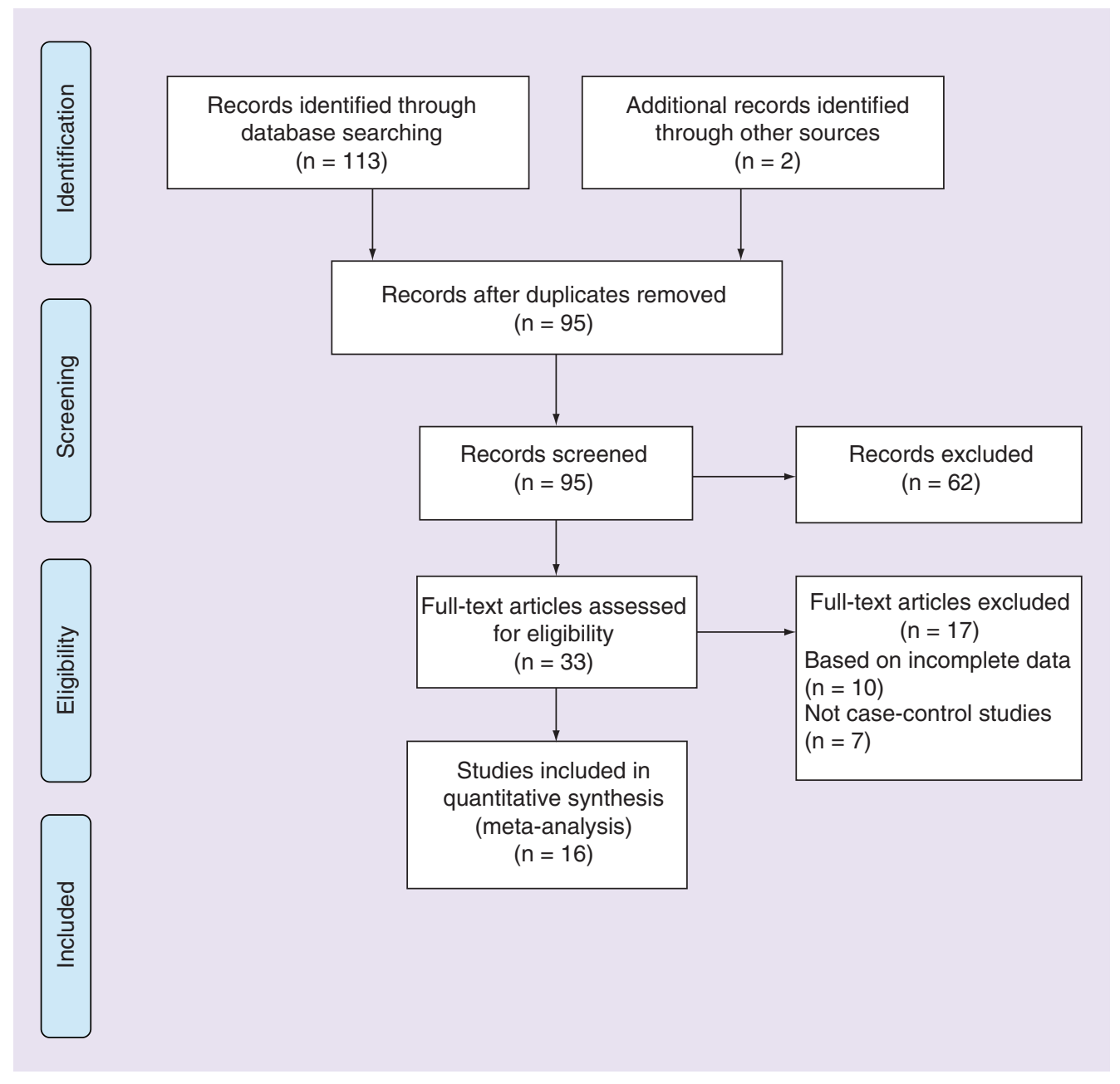

Figure 1. The flow diagram for the included studies.

\section{Sensitivity analysis \& publication bias}

Egger's test and Begg's funnel plot were employed in assessing the publication bias of the selected studies used in our analysis. The symmetrical funnel plot for the various genetic model showed that the outcomes of our analysis were not influenced by bias in publication (Figures $7 \& 8$ ). Furthermore, the outcomes of the Egger's test demonstrated that there was no evidence of bias among our selected studies since the p-values for all the genetic model were higher than 0.05 ( $\mathrm{p}=0.922$ for the recessive model; $\mathrm{p}=0.847$ for the allele model; $\mathrm{p}=0.762$ for the dominant model; $\mathrm{p}=0.740$ for the overdominant model; $\mathrm{p}=0.909$ for the homozygote model). We used sensitivity analysis in the evaluation of our results stability (Figures $9 \& 10$ ). Sensitivity analysis was then investigated to assess the stability of our research results. The statistical significance of the outcomes was not changed when an individual article was omitted, proving the credibility and stability of our research results.

\section{Discussion}

Leukemia is a complicated and multi-factorial disease and for its development, the genetic effect has been considered a significant component [44]. Several research studies have revealed the effects of TP53 Arg72Pro polymorphism on leukemia susceptibility.

Furthermore, experimental studies with transgenic mice confirmed the relationship between TP53 genetic mutation and tumor risk susceptibility and the analysis showed that tumors were developed in mice lacking mutation in one TP53 allele in comparison with the mice in which the mutation was harbored [45]. Also, the polymorphism of TP53 Arg72Pro is the most investigated single nucleotide polymorphism associated with tumor risk. Hence, it is scientifically valid and biologically conceivable for this polymorphism to be associated with the 


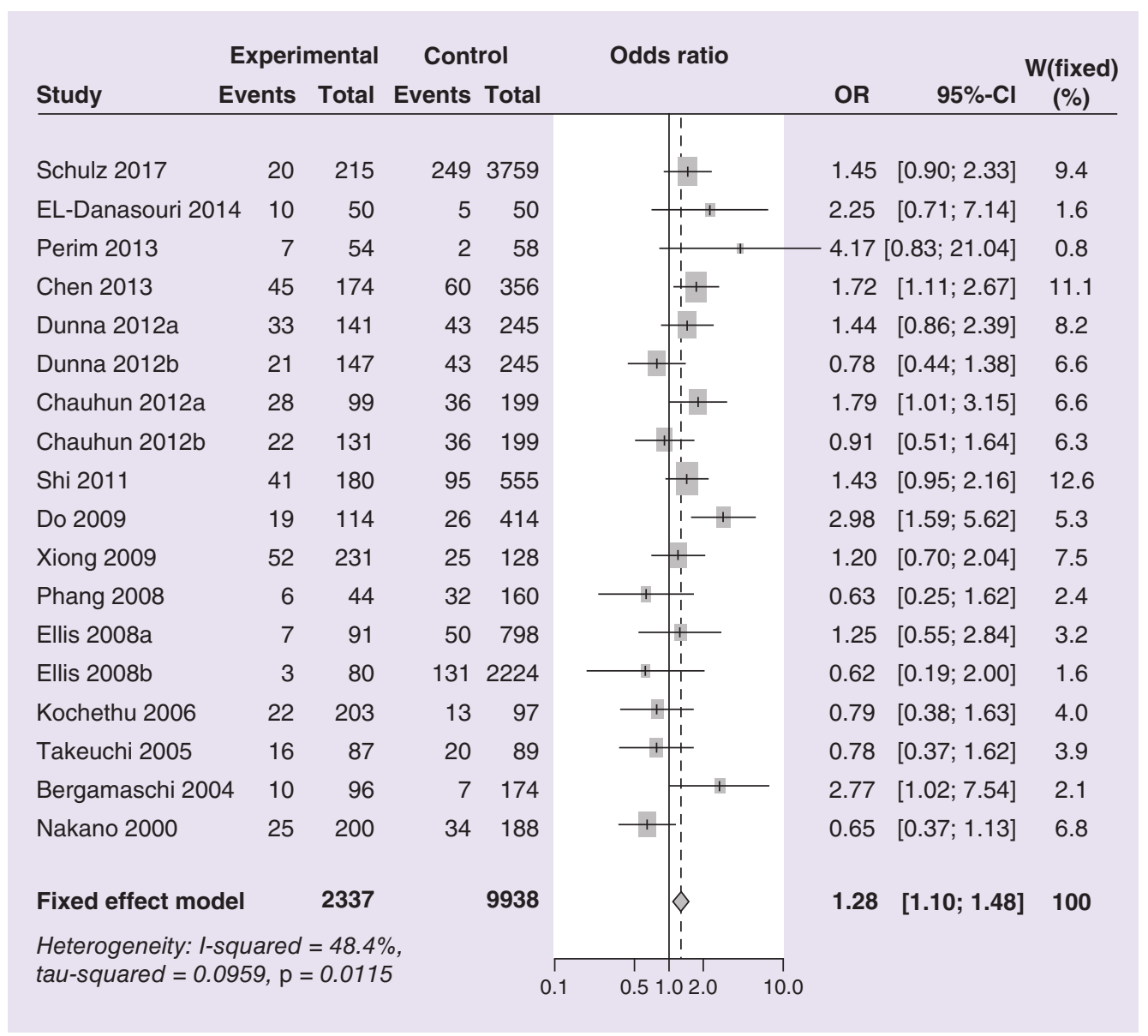

Figure 2. Allele model (C vs G).

development of leukemia. Nevertheless, past meta-analysis studies by Ruan et al. and Tian $e t$ al. [46,47] concluded that there was no relationship between the development of leukemia and TP53 Arg72Pro polymorphism, even when the type of leukemia and ethnicity was considered in their subgroup analysis. The difference between Ruan et al. and Tian et al. studies and our results may be due to different genotype frequencies for polymorphisms in different ethnic groups and differences in sample sizes; however, further investigation is warranted. In this currently updated meta-analysis study with the largest sample size compared with previous studies and specialized software (MetaGenyo) [30] for genome-wide association studies meta-analysis, we have reached some satisfying conclusion. As per our knowledge, our study is the first meta-analysis on the relationship between TP53 Arg72Pro polymorphism and leukemia in a multi-ethnic group.

A total of 18 individual studies with 2337 cases were involved in the analysis. The summary of the overall results of our analysis demonstrated evidence of a significant relationship between Arg72Pro polymorphism of TP53 and leukemia under the recessive and overdominant model. Our finding was consistent with the study of Wang et al. [48], in which they found an association between thyroid cancer and TP53 Arg72Pro polymorphism under the same genetic models. In stratified studies with ethnicity, there was a significant relationship between leukemia and TP53 Arg72Pro polymorphism in Chinese, Japanese, Indian, American, African and mixed populations, but not Caucasian populations. Our finding is similar to the findings of Tian et al. [49] where there was an association between the cancer risk in Asian populations and TP53 Arg72Pro polymorphism. Zoric et al. [50] also demonstrated that there was a positive association between cancer risk in ethnicity and $p 53$ polymorphism Arg72Pro. The substantial ethnicity differences in Arg72Pro polymorphism may result from a genetic background and some influential environmental conditions such as sunlight.

The tumor protein $p 53$ is a major participant in response to stress and by retaliating to diverse epithets, such as the damage of DNA and metabolic stress, it helps maintain genome stability. The functional mechanism loss of $p 53$ 


\begin{tabular}{|c|c|c|c|c|c|c|c|}
\hline \multirow[b]{2}{*}{ Study } & \multicolumn{2}{|c|}{ Experimental } & \multicolumn{2}{|c|}{ Control } & \multirow[t]{2}{*}{ Odds ratio } & \multirow{2}{*}{$95 \%-\mathrm{Cl}$} & W(random) \\
\hline & Events & Total & Events & Total & & & $(\%)$ \\
\hline Schulz 2017 & 118 & 430 & 1980 & 7518 & & $1.06[0.85 ; 1.32]$ & 7.4 \\
\hline EL-Danasouri 2014 & 40 & 100 & 41 & 100 & & $0.96[0.55 ; 1.69]$ & 3.3 \\
\hline Perim 2013 & 37 & 108 & 23 & 116 & + & $-2.11[1.15 ; 3.86]$ & 3.0 \\
\hline Chen 2013 & 180 & 348 & 303 & 712 & - & $1.45[1.12 ; 1.87]$ & 6.8 \\
\hline Dunna 2012a & 110 & 282 & 209 & 490 & & $0.86[0.64 ; 1.16]$ & 6.2 \\
\hline Dunna 2012b & 109 & 294 & 209 & 490 & & $0.79[0.59 ; 1.07]$ & 6.3 \\
\hline Chauhun 2012a & 99 & 198 & 184 & 398 & - & $1.16[0.83 ; 1.64]$ & 5.6 \\
\hline Chauhun 2012b & 115 & 262 & 184 & 398 & & $0.91[0.66 ; 1.25]$ & 6.0 \\
\hline Shi 2011 & 166 & 360 & 479 & 1110 & & $1.13[0.89 ; 1.43]$ & 7.1 \\
\hline Do 2009 & 83 & 228 & 206 & 828 & 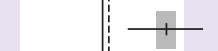 & $1.73[1.26 ; 2.36]$ & 6.0 \\
\hline Xiong 2009 & 231 & 462 & 114 & 256 & & $1.25[0.92 ; 1.69]$ & 6.1 \\
\hline Phang 2008 & 37 & 88 & 136 & 320 & & $0.98[0.61 ; 1.58]$ & 4.1 \\
\hline Ellis $2008 a$ & 45 & 182 & 389 & 1596 & & $1.02[0.71 ; 1.45]$ & 5.4 \\
\hline Ellis $2008 b$ & 41 & 160 & 1100 & 4448 & & $1.05[0.73 ; 1.51]$ & 5.4 \\
\hline Kochethu 2006 & 106 & 406 & 66 & 194 & + & $0.69[0.47 ; 0.99]$ & 5.3 \\
\hline Takeuchi 2005 & 70 & 174 & 77 & 178 & + & $0.88[0.58 ; 1.35]$ & 4.6 \\
\hline Bergamaschi 2004 & 57 & 192 & 75 & 348 & + & $1.54[1.03 ; 2.30]$ & 4.9 \\
\hline Nakano 2000 & 143 & 400 & 163 & 376 & + & $0.73[0.54 ; 0.97]$ & 6.4 \\
\hline Random effects $\mathrm{m}$ & nodel & 4674 & & 19,876 & $>$ & $1.06[0.94 ; 1.21]$ & 100 \\
\hline \multicolumn{3}{|c|}{$\begin{array}{c}\text { Heterogeneity: } \text { I-squared }=61.5 \% \\
\text { tau-squared }=0.044, p=0.0003\end{array}$} & & & $\begin{array}{lll} & & \\
0.5 & 1.0 & 2.0\end{array}$ & & \\
\hline
\end{tabular}

Figure 3. Recessive model (CC vs GC + GG).

is well established in influencing checkpoint controls of the cell cycle and programmed cell death. It also regulates other crucial phases of metastatic development, like invasion and cell migration. The Arg72Pro polymorphism of TP53 Arg72 variant is much more productive in terms of mitochondrial location than the variant of Pro72 and has a greater programmed cell death inducing capability [51]. Researchers discovered that the form of Arg72 activates programmed cell death with quicker kinetics than the variant of Pro72 [52]. The Arg72 protein's higher apoptotic capability originates from this protein's greater interaction with MDM2, promoting nuclear exportation [53]. These two TP53 polymorphism variants are conceptually distinctly different and these differences can affect the risk or treatment of cancer.

Our recent analysis indicates that the Arg72Pro polymorphism of TP53 is related to a higher risk of developing leukemia in Chinese, Indian, Japanese, American, African and mixed populations in line with their biological relationship. Some studies have demonstrated a strong relationship between TP53 mutation and chemotherapy resistance and low overall survival regardless of the low frequency rate of TP53 in hematological cancers [54,55]. The TP53 codon $72 \mathrm{C}$ allele might also be associated more closely with lymphomagenesis. Again, the TP53 mutational status creates opportunities in developing an efficient therapeutic strategy. This meta-analysis is the first to demonstrated that there is a relationship between Arg72Pro polymorphism of TP53 leukemia in a multi-ethnic population. Nevertheless, because of the small handful of studies published, additional studies involving a large population are required to confirm our conclusions.

Nevertheless, some limitations were present in our study. First, the availability of individual data such as family history, age and environmental conditions could have the accuracy of our subgroup analysis. Second, the Arg72Pro 


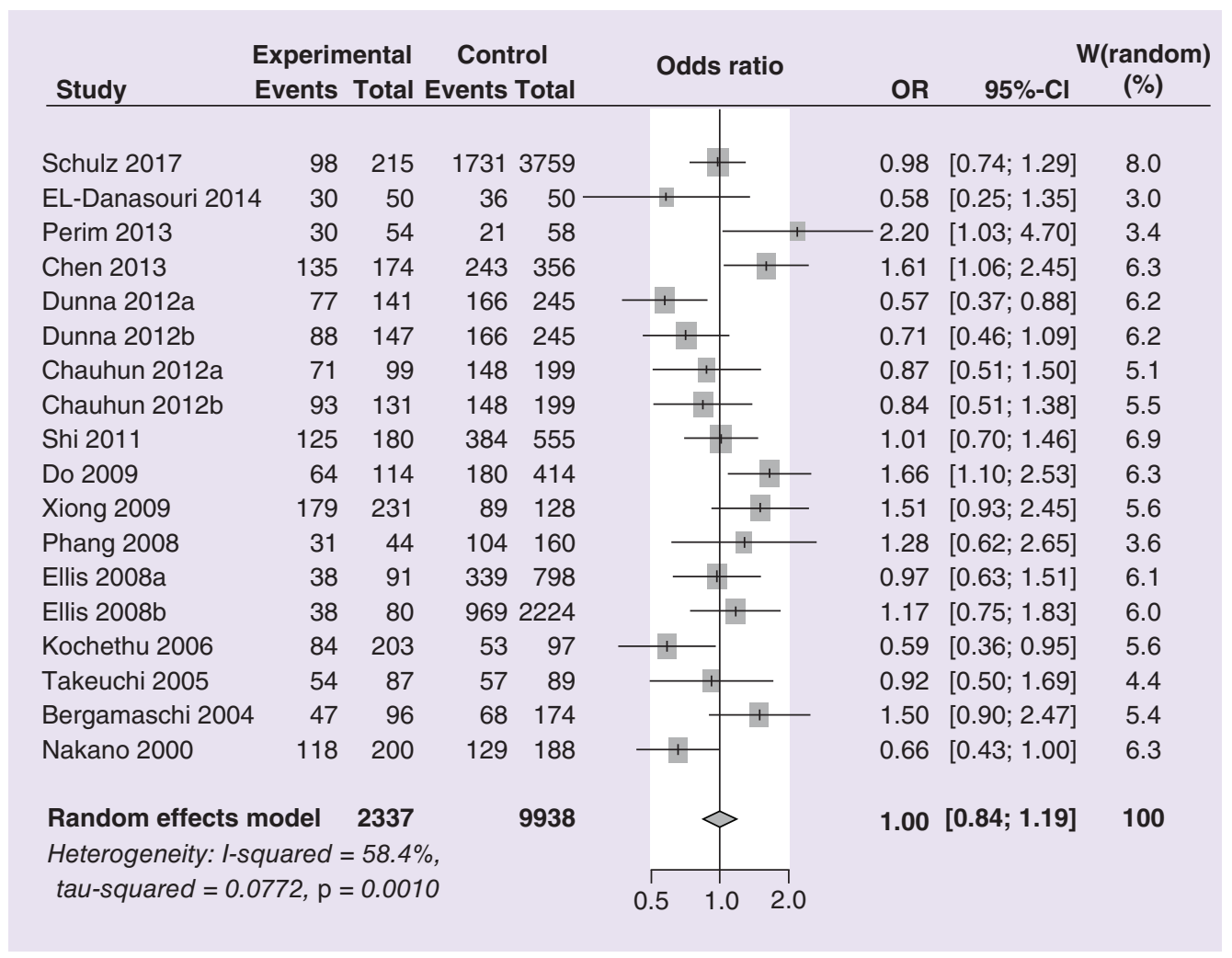

Figure 4. Dominant model (CC + GC vs GG).

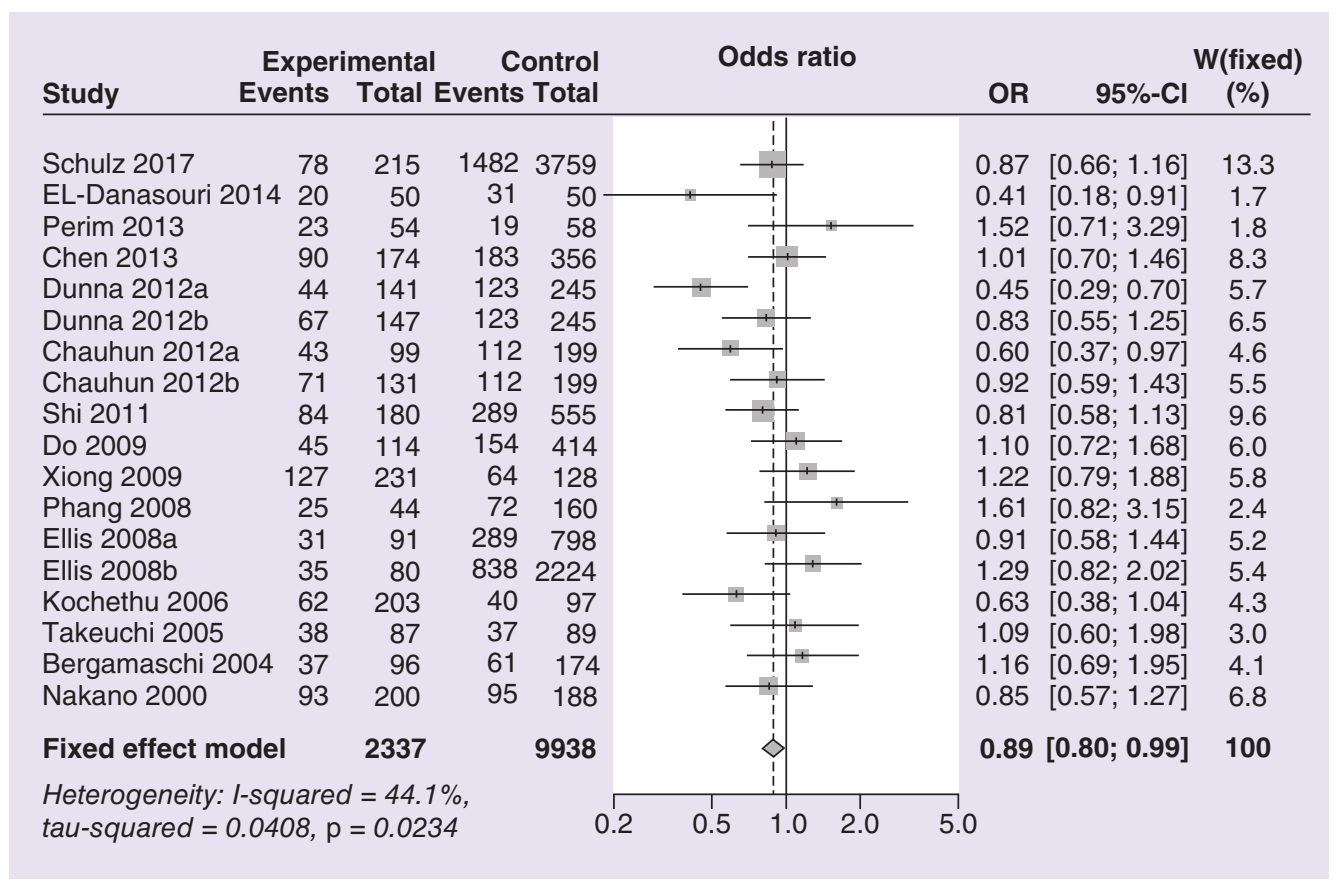

Figure 5. Overdominant model (CG vs CC $+\mathrm{GG}$ ). 


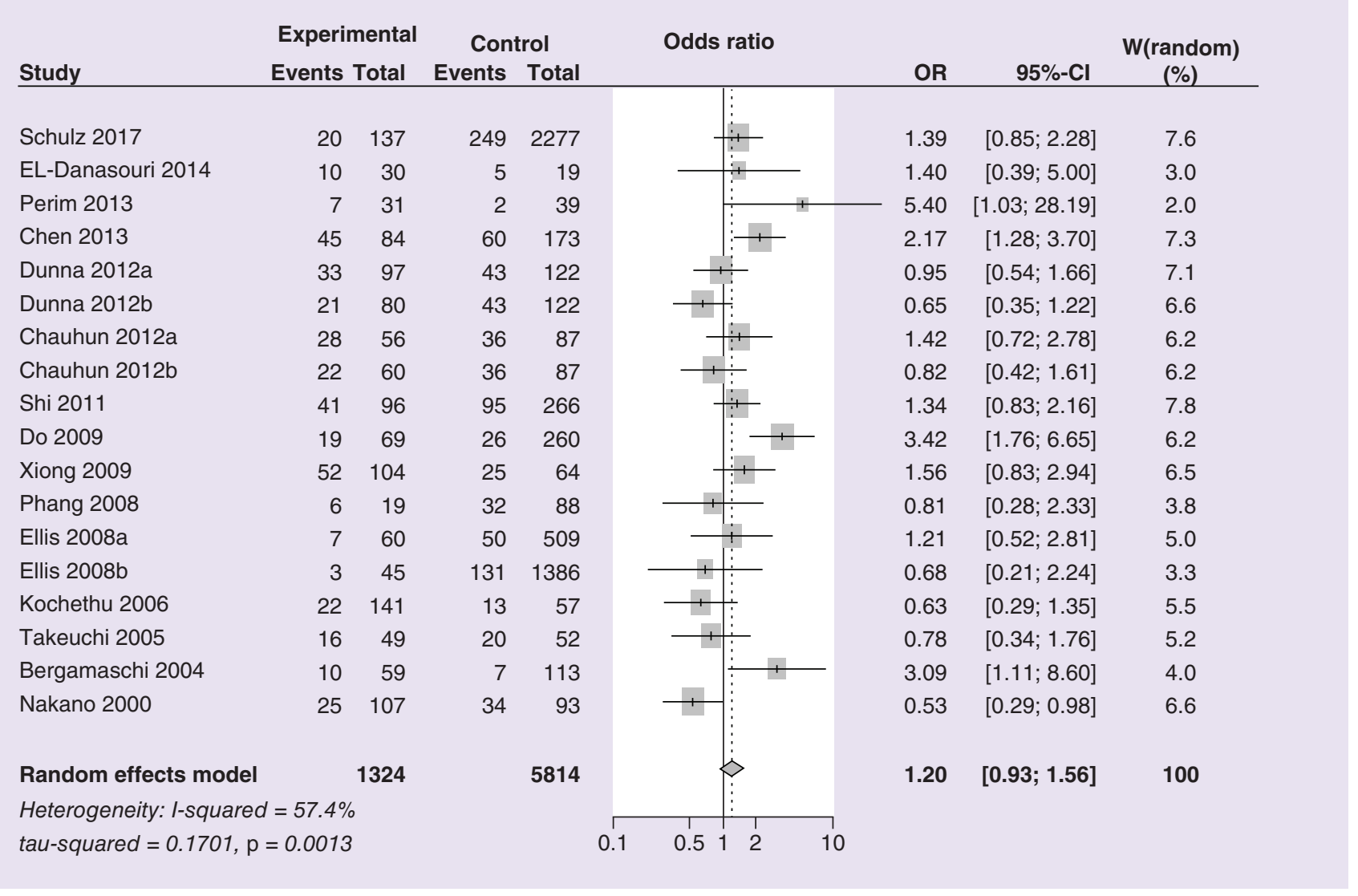

Figure 6. Homozygote model (CC vs GG).

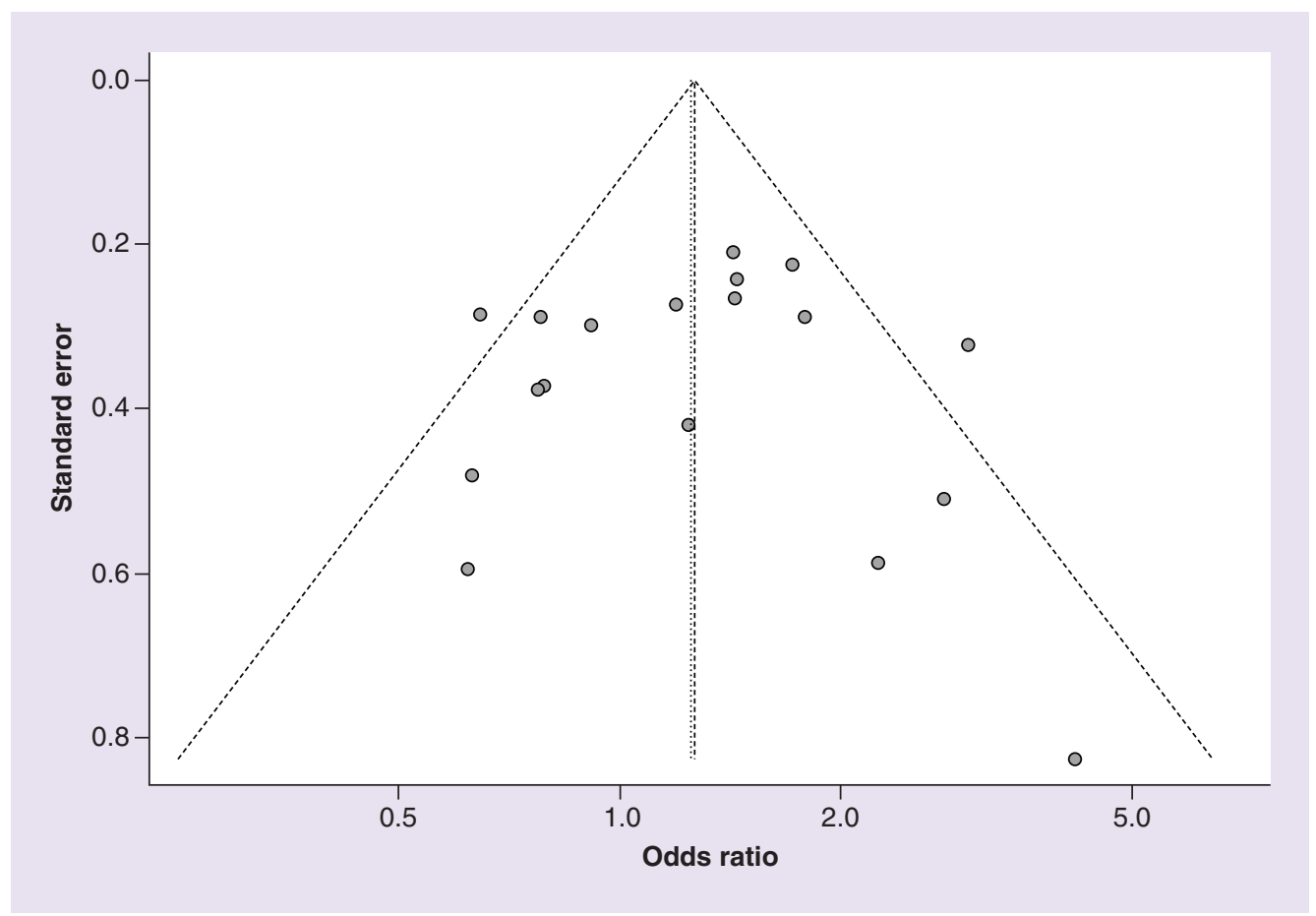

Figure 7. Funnel plot for the recessive model. 


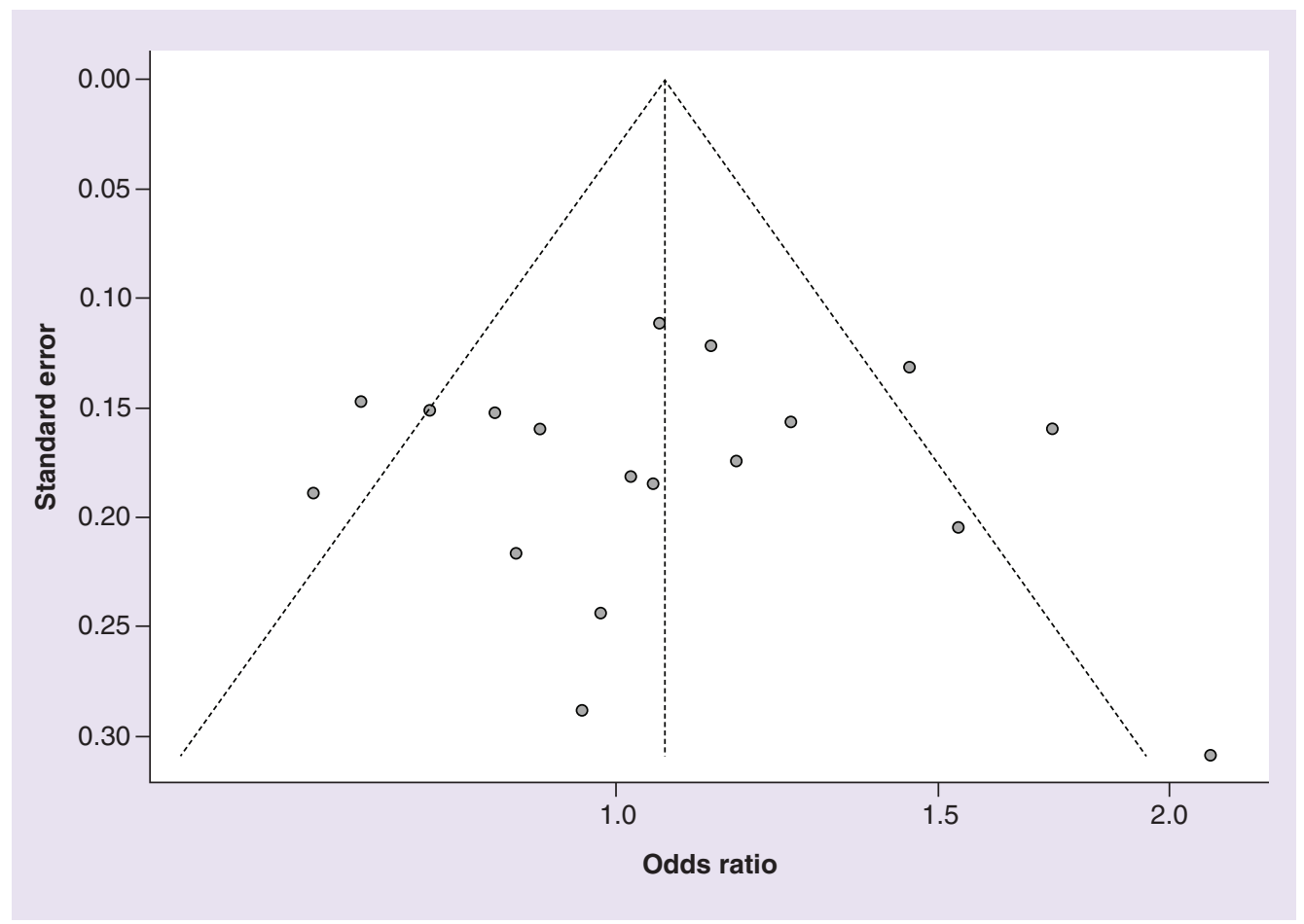

Figure 8. Funnel plot for the allele model.

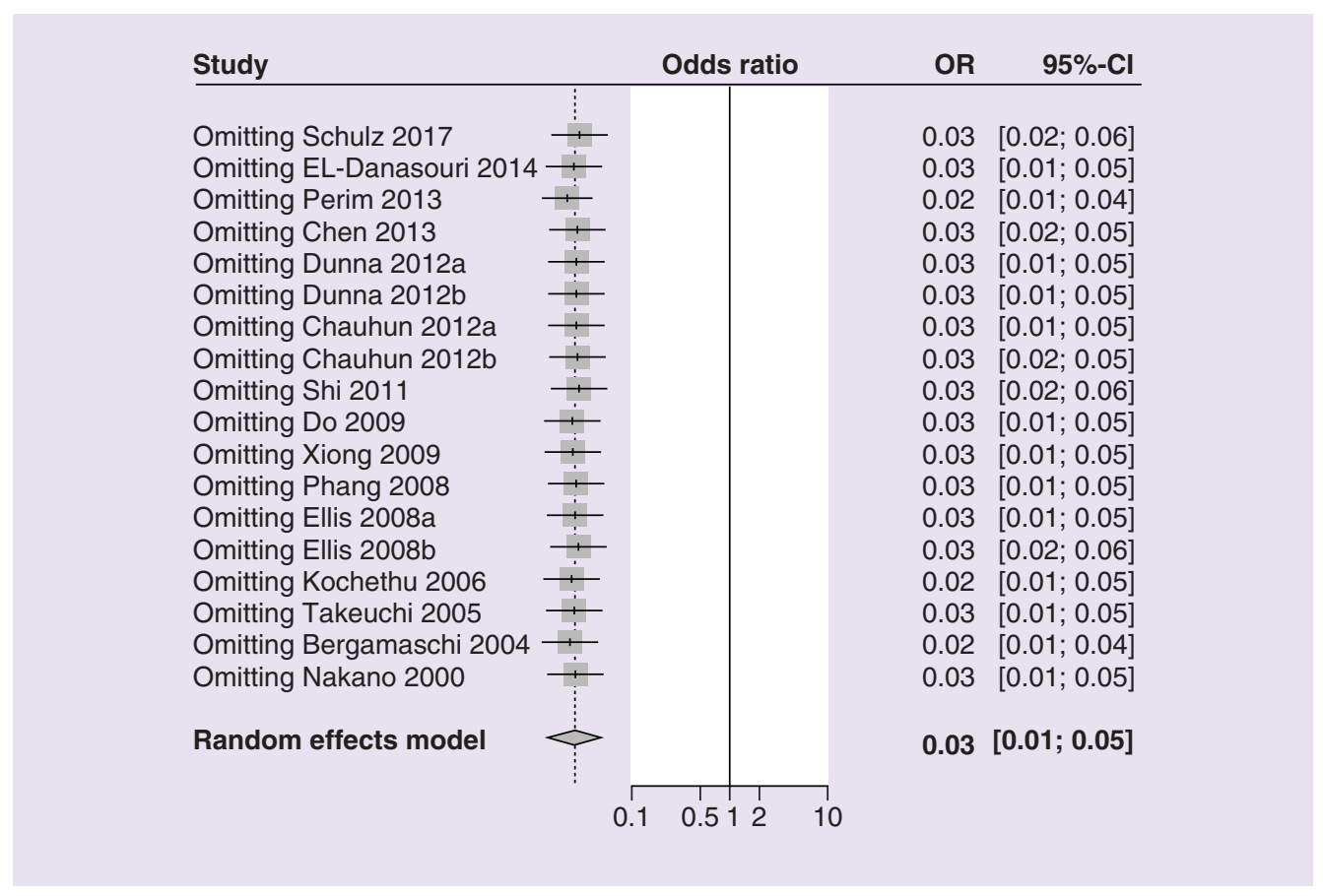

Figure 9. Sensitivity analysis diagram for dominant model.

polymorphism of TP53 may affect leukemia development with other factors like gene-environment and gene-gene interactions, but due to inadequate information, no analysis was done in that aspect. Finally, due to the limited number of published studies, we could not carry out further subgroup stratification analyses by ages. 


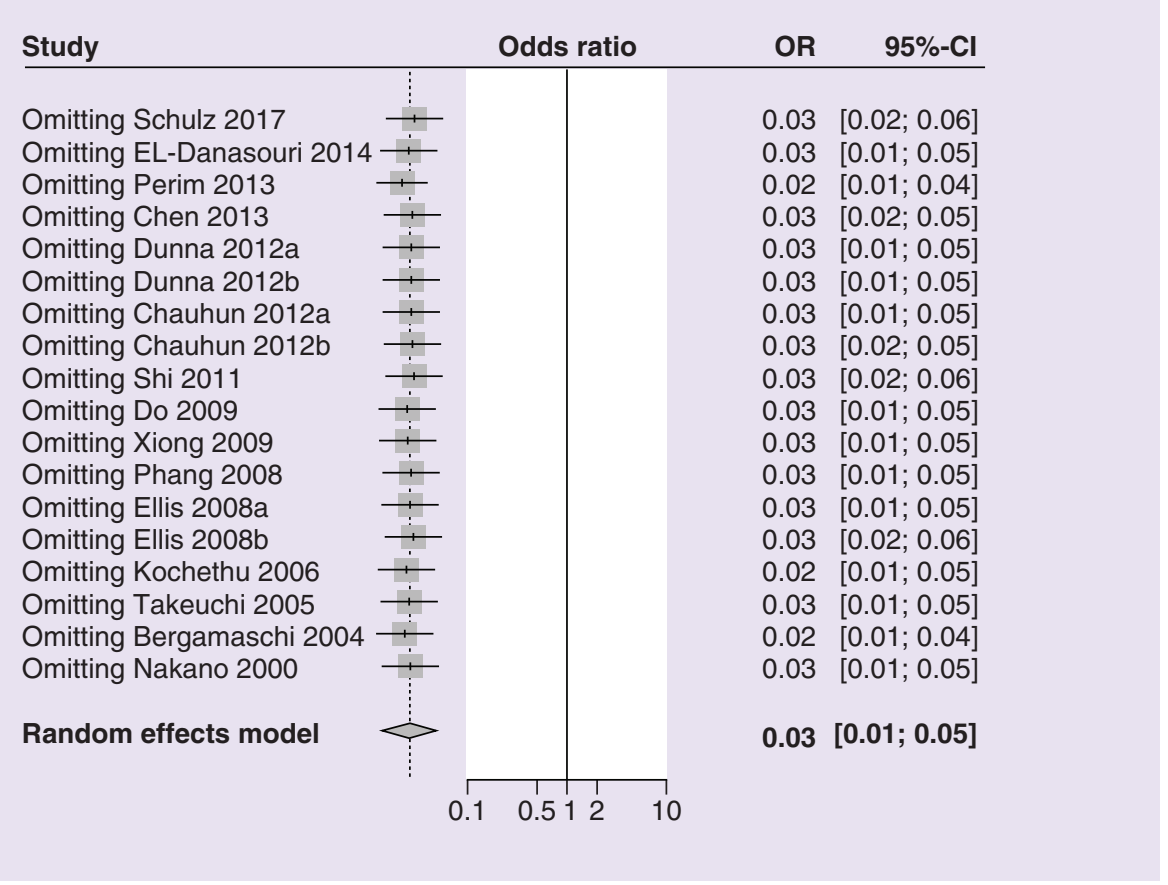

Figure 10. Sensitivity analysis diagram for recessive model.

\section{Conclusion}

We demonstrated that an association exist between leukemia risk and TP53 gene codon Arg72Pro polymorphism in the recessive and overdominant models. Again, our findings show that the TP53 Arg72Pro polymorphism may influence leukemia development in different populations or ethnicities. Analyses with larger samples and a broader ethnic group are required to verify these outcomes.

\section{Summary points}

- Leukemia is a hematopoietic system malignant progressive disease with abnormal or an increased number of immature leucocytes produced by the bone marrow and other blood-forming organs.

- The four major types of leukemia based on cytogenetic analysis are chronic myeloid leukemia, chronic lymphocytic leukemia, acute lymphocytic leukemia and acute myeloid leukemia.

- The $p 53$, also known as tumor protein or TP53, is a gene which codes for a protein that regulates the cell cycle and therefore acts as a tumor suppressor gene.

- P53 has been portrayed (demonstrated) as 'the guardian of the genome' because of its major function in 'conserving stability by preventing genome mutation'.

- The TP53 72Arg variant shows an increased capacity to bring about programmed cell death, while the TP53 72Pro variant brings about the arrest of the cell cycle more efficiently.

- The Arg72 protein's higher apoptotic capability originates from this protein's greater interaction with MDM2, promoting nuclear exportation.

- These two TP53 polymorphism variants are conceptually distinctly different and these differences can affect the risk or treatment of cancer.

- Some studies have demonstrated a strong relationship between TP53 mutation and chemotherapy resistance and low overall survival regardless of the low frequency rate of TP53 in hematological cancers. 
Financial \& competing interests disclosure

This study was partially supported by the National Natural Science Foundation of China (No. 81971508, No. 81273259 and No. 81471589), the Health Bureau of Henan Province, PR China (No. 201201005) and the foundation and frontier research grant of Henan provincial science and technology bureau, PR China (No.112300410027 and No. 132102310120). The authors have no other relevant affiliations or financial involvement with any organization or entity with a financial interest in or financial conflict with the subject matter or materials discussed in the manuscript.

No writing assistance was utilized in the production of this manuscript.

Availability of data

The data analyzed for the current study is available with the corresponding author and can be released on reasonable request.

\section{Open access}

This work is licensed under the Attribution-NonCommercial-NoDerivatives 4.0 Unported License. To view a copy of this license, visit http://creativecommons.org/licenses/by-nc-nd/4.0/

\section{Reference}

Papers of special note have been highlighted as: $\bullet$ of interest;

1. Estey EH. Prognostic factors in acute myelogenous leukemia. Leukemia 15(4), 670-672 (2001).

2. Satoh Y, Matsumura I, Tanaka $\mathrm{H}$ et al. C-terminal mutation of RUNX1 attenuates the DNA-damage repair response in hematopoietic stem cells. Leukemia 26 (2), 303-311 (2012).

- Provides an overview required for multi-step leukemogenesis and the genomic instability caused by impaired Gadd45a function.

3. Drokow EK, Sun K, Ahmed HA, Akpabla GS, Song J, Shi M. Circulating microRNA as diagnostic biomarkers for haematological cancers: a systematic review and meta-analysis. Cancer Manag. Res. 11, 4313 (2019).

4. Li SY, Ye JY, Liang EY, Zhou LX, Yang M. Association between MTHFR C677T polymorphism and risk of acute lymphoblastic leukemia: a meta-analysis based on 51 case-control studies. Med. Sci. Monit. 21, 740 (2015).

5. Chen J, Liu Y, Cai QQ et al. Type D personality parents of children with leukemia tend to experience anxiety: the mediating effects of social support and coping style. Medicine 94.10 (2015).

6. Kowalczyk J, Nurzynska-Flak J, Armata J et al. Incidence and clinical characteristics of second malignant neoplasms in children: a multicenter study of a polish pediatric leukemia/lymphoma group. Med Sci. Monit. 10(3), CR117-CR122 (2004).

7. Vardiman JW, Thiele J, Arber DA et al. The 2008 revision of the World Health Organization (WHO) classification of myeloid neoplasms and acute leukemia: rationale and important changes. Blood 114(5), 937-951 (2009).

8. Ma Z, Morris SW, Valentine V et al. Fusion of two novel genes, RBM15 and MKL1, in the $\mathrm{t}(1 ; 22)$ (p13; q13) of acute megakaryoblastic leukemia. Nat. Genet. 28(3), 220-221 (2001).

9. Weng Y, Lu L, Yuan G et al. p53 codon 72 polymorphism and hematological cancer risk: an update meta-analysis. PLoS ONE 7(9), e45820 (2012).

10. Descatha A, Jenabian A, Conso F, Ameille J. Occupational exposures and haematological malignancies: overview on human recent data. Cancer Causes Control 16(8), 939-953 (2005).

11. Brosh R, Rotter V. When mutants gain new powers: news from the mutant $p 53$ field. Nat. Rev. Cancer 9(10), 701-713 (2009).

12. Hainaut P, Wiman KG. 30 years and a long way into p53 research. Lancet Oncol. 10 (9), 913-919 (2009).

- Discusses the progress and understanding of $p 53$ from obscure oncogene to key tumor-suppressor gene with clinical potential.

13. Francisco G, Menezes PR, Eluf-Neto J, Chammas R. Arg72Pro TP53 polymorphism and cancer susceptibility: a comprehensive meta-analysis of 302 case-control studies. Int. J. Cancer 129(4), 920-930 (2011).

14. Srivastava S, Zou Z, Pirollo K, Blattner W, Chang EH. Germline transmission of a mutated p 53 gene in a cancer-prone family with Li-Fraumeni syndrome. Nature 348 (6303), 747-749.

15. Barbosa K, Li S, Adams PD, Deshpande AJ. The role of TP53 in acute myeloid leukemia: challenges and opportunities. Genes Chromosomes Cancer 58 (12), 875-888 (2019).

- This review suggested the use of pharmacological activators of TP53 and it clinical benefit in acute myeloid leukaemia.

16. Malkin D, Nichols KE, Zelley K, Schiffman JD. Predisposition to pediatric and hematologic cancers: a moving target. Am. Soc. Clin. Oncol. Educ. B 34(1), e44-e55 (2014).

17. Cancer Genome Atlas Research Network. Genomic and epigenomic landscapes of adult de novo acute myeloid leukemia the cancer genome atlas research network. N. Engl. J. Med. 368(22), 2059-2074 (2013).

18. Papaemmanuil E, Gerstung M, Bullinger L et al. Genomic classification and prognosis in acute myeloid leukemia. N. Engl. J. Med. 374(23), 2209-2221 (2016). 
19. Quintás-Cardama A, Hu C, Qutub A et al. P53 pathway dysfunction is highly prevalent in acute myeloid leukemia independent of TP53 mutational status. Leukemia 31(6), 1296-1305 (2017).

20. Welch JS, Petti AA, Miller CA et al. TP53 and decitabine in acute myeloid leukemia and myelodysplastic syndromes. N. Engl. J. Med. 375(21), 2023-2036 (2016).

21. Pant V, Quintás-Cardama A, Lozano G. The p53 pathway in hematopoiesis: lessons from mouse models, implications for humans. Blood 120(26), 5118-5127 (2012).

22. Stoddart A, Fernald AA, Wang J et al. Haploinsufficiency of del (5q) genes, Egr1 and Apc, cooperate with Tp53 loss to induce acute myeloid leukemia in mice. Blood 123(7), 1069-1078 (2014).

23. McGraw KL, Zhang LM, Rollison DE et al. The relationship of TP53 R72P polymorphism to disease outcome and TP53 mutation in myelodysplastic syndromes. Blood Cancer J. 5(3), e291-e291 (2015).

24. McGraw KL, Cluzeau T, Sallman DA et al. TP53 and MDM2 single nucleotide polymorphisms influence survival in non-del (5q) myelodysplastic syndromes. Oncotarget 6(33), 34437 (2015).

- A useful research article discussing the role of TP53 in preleukemia conditions.

25. Pim D, Banks L. p53 polymorphic variants at codon 72 exert different effects on cell cycle progression. Int. J. Cancer 108(2), 196-199 (2004).

26. Ara S, Lee PS, Hansen MF, Saya H. Codon 72 polymorphism of the TP53 gene. Nucleic Acids Res. 18(16), 4961 (1990).

27. Bergamaschi G, Merante S, Orlandi E, Galli A, Bernasconi P, Cazzola M. TP53 codon 72 polymorphism in patients with chronic myeloid leukemia. Haematologica 89(7), 868-869 (2004).

28. Ruan XL, Li S, Zeng XT, Xia LH, Hu Y. No association between cytochrome P450 2D6 gene polymorphism and risk of acute leukemia: evidence based on a meta- analysis. Chin. Med. J. (Engl.) 126(), 3750-3753 (2013).

29. Egger M, Davey Smith G, Schneider M, Minder C. Bias in meta- analysis detected by a simple, graphical test. BMJ 315(7109), 629-634 (1997).

30. Martorell-Marugan J, Toro-Dominguez D, Alarcon-Riquelme ME, Carmona-Saez P. MetaGenyo: a web tool for meta-analysis of genetic association studies. BMC Bioinformatics 18(1), 563 (2017).

31. Schulz E, Lind K, Renner W et al. The TP53 Pro72Arg SNP in de novo acute myeloid leukemia - results of two cohort studies involving 215 patients and 3759 controls. Br. J. Haematol. 181(1), 148 (2018).

32. El-Danasouri NM, Ragab SH, Rasheed MA, El_Saadany ZA, Abd El-Fattah SN. MDM2 SNP309 and p53 codon 72 genetic polymorphisms and risk of AML: an Egyptian study. Ann. Clin. Lab. Sci. 44(4), 449-454 (2014).

33. de Lourdes Perim A, Guembarovski RL, Oda JM et al. CXCL12 and TP53 genetic polymorphisms as markers of susceptibility in a Brazilian children population with acute lymphoblastic leukemia (ALL). Mol. Biol. Rep. 40 (7), 4591-4596 (2013).

34. Chen J, Zhu B, Chen J, Li Y. Genetic variations in MDM2 and P53 genes confer risk for adult acute lymphoblastic leukemia in a Chinese population. DNA Cell Biol. 32 (7), 414-419 (2013).

35. Chauhan PS, Ihsan R, Mishra AK et al. High order interactions of xenobiotic metabolising genes and P53 codon 72 polymorphisms in acute leukemia. Environ. Mol. Mutagen. 53(8), 619-630 (2012).

36. Shi JY, Ren ZH, Jiao B et al. Genetic variations of DNA repair genes and their prognostic significance in patients with acute myeloid leukemia. Int. J. Cancer 128(1), 233-238 (2011).

37. Do TN, Ucisik-Akkaya E, Davis CF, Morrison BA, Dorak MT. TP53 R72P and MDM2 SNP309 polymorphisms in modification of childhood acute lymphoblastic leukemia susceptibility. Cancer Genet. Cytogenet. 195(1), 31-36 (2009).

38. Xiong X, Wang M, Wang L et al. Risk of MDM2 SNP309 alone or in combination with the $\mathrm{p} 53$ codon 72 polymorphism in acute myeloid leukemia. Leuk. Res. 33(11), 1454-1458 (2009).

39. Phang BH, Linn YC, Li H, Sabapathy K. MDM2 SNP309 G allele decreases risk but does not affect onset age or survival of Chinese leukemia patients. Eur. J. Cancer 44(5), 760-766 (2008).

40. Ellis NA, Hou D, Yildiz O et al. MDM2 SNP309 and TP53 Arg72Pro interact to alter therapy-related acute myeloid leukemia susceptibility. Blood 112(3), 741-749 (2008).

41. Kochethu G, Delgado J, Pepper C et al. Two germ line polymorphisms of the tumour suppressor gene p53 may influence the biology of chronic lymphocytic leukemia. Leuk. Res. 30(9), 1113-1118 (2006).

42. Takeuchi S, Matsushita M, Tsukasaki $\mathrm{K}$ et al. $\mathrm{P} 53$ codon 72 polymorphism is associated with disease progression in adult T-cell leukemia/lymphoma. Br. J. Haematol. 131(4), 552-553 (2005).

43. Nakano Y, Naoe T, Kiyoi H et al. Poor clinical significance of $p 53$ gene polymorphism in acute myeloid leukemia. Leuk. Res. 24(4), 349-352 (2000).

44. Legrand F, Renneville A, Macintyre E et al. The spectrum of FIP1L1-PDGFRA-associated chronic eosinophilic leukemia: new Insights based on a survey of 44 cases. Medicine 92(5), 273-284 (2013).

45. Donehower LA, Harvey M, Slagle BL et al. Mice deficient for $p 53$ are developmentally normal but susceptible to spontaneous tumours. Nature 356(6366), 215-221 (1992). 
46. Ruan XL, Li S, Meng XY, Geng P, Gao QP, Ao XB. The role of TP53 gene codon 72 polymorphism in leukemia: a PRISMA-compliant systematic review and meta-analysis. Medicine 94(38), e1588 (2015).

47. Tian X, Dai S, Sun J, Jiang Y. Association between TP53 Arg72Pro polymorphism and leukemia risk: a meta-analysis of 14 case-control studies. Sci. Rep. 6(1), 24097 (2016).

48. Wang F, Wang P, Wang B et al. Association between TP53 Arg72Pro polymorphism and thyroid carcinoma risk. Tumor Biol. 35(3), 2723-2728 (2014).

49. Tian X, Dai S, Sun J, Jiang S, Jiang Y. The association between the TP53 Arg72Pro polymorphism and colorectal cancer: an updated meta-analysis based on 32 studies. Oncotarget. 8(1), 1156-1165 (2017).

50. Zorić A, Horvat A, Balija M, Slade N. The Arg72Pro polymorphism of TP53 in Croatian population. Croat. Chem. Acta 85 (2), 239-243 (2012).

51. Dumont P, Leu JI, Della Pietra AC, George DL, Murphy M. The codon 72 polymorphic variants of p 53 have markedly different apoptotic potential. Nat. Genet. 33(3), 357-365 (2003).

52. Thomas M, Kalita A, Labrecque S, Pim D, Banks L, Matlashewski G. Two polymorphic variants of wild-type p53 differ biochemically and biologically. Mol. Cell. Biol. 19(2), 1092-1100 (1992).

53. Boyd SD, Tsai KY, Jacks T. An intact HDM2 RING- finger domain is required for nuclear exclusion of p53. Nat. Cell Biol. 2(9), 563-568 (2000).

54. Preudhomme C, Fenaux P. The clinical significance of mutations of the P53 tumour suppressor gene in haematological malignancies. Br. J. Haematol. 98(3), 502-511 (1997).

55. Krug U, Ganser A, Koeffler HP. Tumor suppressor genes in normal and malignant hematopoiesis. Oncogene 21(21), 3475-3495 (2002). 
